\title{
Relación entre desigualdad y crimen en el noreste de México
}

\author{
Relationship between inequality and crime in Northeast Mexico
}

Luis Lauro Carrillo-Sagástegui ${ }^{1}$, Francisco García-Fernández ${ }^{2}$

Fecha de recepción: 18 de octubre de 2020

Fecha de aceptación: 25 de enero de 2021

1- Nacionalidad: Mexicana. Grado: Maestría en Política y Gobierno. Estudiante del Doctorado en Ciencias Administrativas bajo la línea de investigación de Desarrollo Regional. Adscripción: Universidad Autónoma de Tamaulipas. ORCID: https://orcid.org/0000-0001-8354-5791. Correo electrónico: carrillo.sagastegui@gmail.com

2- Nacionalidad: Mexicana. Grado: Doctorado. Adscripción: Universidad Autónoma de Tamaulipas. ORCID: https:// orcid.org/0000-0003-4340-1093. Correo electrónico: ffernandez@docentes.uat.edu.mx 


\section{Resumen}

La desigualdad y el crimen son fenómenos que atentan contra la calidad de vida de los ciudadanos. El presente estudio tiene como objetivos i) analizar la relación entre desigualdad y la tasa de delitos por cada 100 habitantes en áreas geoestadísticas básicas del municipio de Victoria, México; ii) determinar la capacidad predictiva que tiene la desigualdad sobre el crimen; $y$ iii) identificar qué delitos se correlacionan más con desigualdad. Se asume que existe una relación positiva entre el coeficiente de Gini y la tasa de delitos. Se aplicó un modelo de regresión lineal simple. Los resultados indican una correlación ( $r=$ $0.510)$ significante $(P>0.001)$, y la varianza explicada $(r \wedge 2=0.26)$; así mismo, el modelo señala que por cada grado que aumenta la desigualdad aumentan 1.114 delitos por cada 100 habitantes; $y$ el robo domiciliario es el crimen que más se correlaciona con la desigualdad con $(r=0.376)$.

Palabras clave: desigualdad, bienestar, criminalidad, municipio, AGEB, espacio urbano, noreste de México

\section{Abstract}

Inequality and crime are phenomena that threaten the quality of life of citizens. This study aims to i) analyze the relationship between inequality and the crime rate per 100 inhabitants in Basic Geostatistical Areas of the municipality of Victoria, Mexico; ii) determine the predictive capacity of crime inequality; and iii) identify which crimes are most correlated with inequality. It is assumed that there is a positive relationship between the Gini coefficient and the crime rate. A simple linear regression model was applied. The results indicate a significant correlation $(r=0.510)(P>0.001)$, and the explained variance $(r$ $\wedge 2$ =0.26); Likewise, the model indicates that for each degree that inequality increases, 1,114 crimes per 100 inhabitants increase; and house robbery is the crime that most correlates with inequality, with $(r=0.376)$.

Keywords: inequality, wealth, criminality, municipality, basic geostatistical areas, urban space, northeast of Mexico 


\section{Introducción}

$\mathrm{L}$

os problemas de violencia y criminalidad no son fenómenos recientes de la historia de México. Han acompañado la historia del país desde antes de la conquista. Todo el siglo XX fue testigo de acciones violentas continuadas, concentradas en distintas regiones del país. Con el auge del narcotráfico y el enfrentamiento público durante el gobierno de Felipe Calderón (20062012), hay un punto de inflexión, pues tiene lugar una espiral de crimen y violencia que abarca casi todo México, aunque con mayor énfasis en un grupo de estados, entre ellos los de la frontera norte del país, como Tamaulipas. Ninguno de sus municipios fue ajeno a esta problemática. En particular, el municipio de Victoria, que abarca la ciudad capital, que también ha estado sometida al incremento del crimen en general. La ubicación geográfica del municipio, en el mismo centro del estado y su cercanía a la frontera norte con los Estados Unidos, lo colocan en una posición geográfica ideal en la ruta de los productos del narcotráfico (Correa-Cabrera, 2015; Vásquez Galán y Corrales Corrales, 2017).

Si bien este es un fenómeno con larga historia en México, los estudios que abordan este problema, desde una perspectiva científica, son relativamente recientes. En particular, las investigaciones que se orientan a identificar los problemas con los cuales puede estar relacionado ese fenómeno. Este trabajo se enfoca en la desigualdad económica, como factor que facilita la proliferación del crimen.

La relación entre crimen y desigualdad ha sido estudiada desde diferentes enfoques de las Ciencias Sociales y del comportamiento humano, interesadas en hallar alguna relación entre ambos. Una de las perspectivas ha estudiado el crimen como un resultado del desajuste en las variables del desarrollo, pues la incertidumbre en el retorno esperado genera una reducción a corto plazo de las decisiones de inversión (Detotto y Otranto, 2010). Otros la consideran como producto de un diseño deficiente de la capacidad redistributiva del estado (Danziger y Wheeler, 1975). Otras perspectivas asocian la criminalidad y su incremento en determinados periodos históricos, a un conjunto de factores de índole económica y social.

Para el caso de México, un estudio relativamente reciente de Enamorado et al. (2016) halló que la desigualdad tiende a propiciar decisiones individuales antisociales, en condiciones de falta de empleo e impunidad. Por otra parte, un enfoque más amplio encontró que la relación entre violencia y desigualdad puede reforzarse, derivada de la desorganización social y la disminución de capital social, resultando una dinámica negativa dentro de las comunidades (Kennedy et al., 1998; Kramer, 2000).

En los estudios precedentes, los investigadores trabajaron sobre diversos datos agregados y desagregados, con un enfoque macro o micro, algunos basando el nivel de análisis en datos recopilados en encuestas, otros con datos secundarios. En México, no se han encontrado estudios previos que aborden la relación entre crimen y desigualdad a un nivel desagregado con información de AGEB, como describe este trabajo. El presente estudio, con este nivel de desagregación, sería un aporte a la investigación de estos fenómenos, aunque también significa un reto, debido a la complejidad y dificultad por la escasa disponibilidad de datos. Por último, con la información obtenida de una localidad específica (Victoria, Tamaulipas), se pretende contribuir al diseño e implementación de las políticas públicas del municipio. 
El presente trabajo tiene como propósito analizar la relación existente entre crimen y desigualdad económica en el contexto municipal de Victoria, Tamaulipas. Para ello, se desarrolló la siguiente estructura: introducción, revisión de los antecedentes teóricos y empíricos, metodología utilizada, variables construidas, el análisis, la discusión de los resultados y, por último, se presentan las conclusiones.

\section{En este trabajo, se propone responder las siguientes preguntas de investigación:}

(1) ¿La desigualdad económica está correlacionada con el aumento del crimen en el municipio de Victoria?

(2)¿Qué capacidad predictiva tiene la desigualdad por ingreso sobre el crimen?

(3)¿Qué delitos están más correlacionados con la desigualdad económica?

\section{La relación entre desigualdad y crimen: antecedentes teóricos y empíricos}

La relación entre desigualdad y crimen ha sido investigada desde varias perspectivas teóricas. Las más importantes son: Becker (1968) con la teoría económica del crimen, Merton (1938) con la teoría de la frustración, por último, Shaw y McKay (1942) con la teoría de la desorganización social.

Becker (1968), establece una lógica económica del crimen al considerar al actor como persona en un contexto de desigualdad. Desde la perspectiva neoclásica de la economía, parte del supuesto de la racionalidad del individuo y de que su toma de decisiones se basa en la relación costo-beneficio. Un individuo comete un delito si la utilidad esperada del mismo supera el beneficio que obtendría utilizando su tiempo y recursos en actividades lícitas. Es decir, los individuos asignan su tiempo entre actividades lícitas de mercado y las no lícitas o actividades criminales, en función de los rendimientos esperados y de dos factores: la probabilidad de condena por delitos cometidos y la severidad de la pena a cumplir.

Desde una perspectiva sociológica, Merton (1938) defiende una llamada teoría de la frustración, según la cual los individuos en condiciones de precariedad desarrollan emociones de frustración por no tener acceso a los bienes materiales y al éxito de aquellos que se encuentran en grupos sociales favorecidos. Según el autor, un individuo pobre es más probable que delinca en un lugar con desigualdad elevada, que en lugares donde la desigualdad es baja. Para Merton (1938), las emociones dictadas por la frustración son las que conducen a cometer actividades delictivas.

A diferencia de Becker (1968), Shaw y McKay (1942) consideran factores de índole social y cultural en su teoría de la desorganización social. Entre esos factores están la pobreza, heterogeneidad étnica y la movilidad residencial, las cuales contribuyen a debilitar la efectividad de los mecanismos de control social. Según Shaw y Mckay (1948) los factores mencionados afectan el papel de las redes de control social y la capacidad de las familias y comunidades para ejercer un control efectivo e informal sobre sus miembros. Desde esa perspectiva, estos autores consideran que la desigualdad está relacionada con la pobreza y en esa medida es factor determinante de la criminalidad.

En estudios realizados en Estados Unidos y en Europa, se ha encontrado una relación positiva y robusta entre pobreza y delincuencia (Pridemore, 2011). Sin embargo, en las investigaciones 
sobre la relación entre desigualdad y crimen, los resultados han sido diversos y no tan convincentes (Pridemore y Trent, 2010; Pridemore, 2011).

Ehrlich (1973); Blau y Blau (1982); Kelly (2000); Fajnzylber et al. (2002); Lee et al. (2014); Coccia (2017); Costantini et al. (2018) encontraron una relación positiva entre desigualdad y nivel de homicidios, robos, ataques a la propiedad, entre otros. En cambio, otros autores no detectaron una relación positiva clara (Neumayer, 2005; Pridemore, 2011). Por ejemplo, el trabajo de Chintrakarn y Herzer (2012) obtuvo en un estudio realizado a nivel nacional en Estados Unidos, con base en una muestra de 2,091 observaciones en el periodo de 1965-2005, resultados negativos: a mayores niveles de desigualdad, menor crimen.

En trabajos con análisis desagregados a nivel de vecindario, los resultados son contradictorios. Por un lado, Chamberlain y Hipp (2015) y Metz y Burdina (2018), reportaron una relación positiva entre desigualdad y crimen. En estos estudios, la desigualdad es medida en la interacción entre barrios estadounidenses de mayor ingreso con los de menor ingreso. Por otro lado, Kang (2016) documentó que las relaciones positivas señaladas en reportes nacionales cuando se trasladan a un nivel desagregado, no son del todo claras, el estudio ubica a la concentración de la riqueza como predictor de crimen.

La relación entre desigualdad y crimen ha sido un tema de interés para las Ciencias Sociales y los tomadores de decisiones de política pública. El auge de la violencia y de los delitos criminales en México a partir de 2006-2007, aproximadamente, han sido abordados por una literatura que ha pretendido identificar las causas explicativas de estos fenómenos. Más de una década después, continúan presentes e influyendo la vida cotidiana de los mexicanos en todo el país (Merino, 2011; Hope, 2013; Enamorado et al., 2016). De índole interno, Hope (2013), señala el incremento de los conflictos entre bandas de narcotraficantes por la repartición de territorios a partir del 2006. Hope (2013), en particular considera que el auge de la criminalidad a partir del 2007 está asociado a varias causas, de índole externas e internas. Con relación a las primeras, señala el alza del precio de la droga (cocaína) en los Estados Unidos, mayor cantidad de armamento disponible en México, la disminución de los precios de las armas en ese país y la supresión de la prohibición de la venta de armas de asalto desde el 2004, mayor número de personas con capacidad para delinquir, así como por el aumento de las repatriaciones. Por su parte, Enamorado et al. (2016) examinaron la relación entre desigualdad y crimen en el contexto de la estrategia contra el crimen organizado implementada durante el gobierno de Felipe Calderón. Los autores encontraron una relación positiva e identificaron que cada punto porcentual de incremento en el coeficiente de Gini representa un aumento de seis homicidios por cada 100 mil habitantes, en el periodo de estudio 2007-2010.

Estudios científicos recientes enfocados en los estados del noreste de México (Tamaulipas y Nuevo León), han intentado determinar la relación entre crimen y situación socioeconómica de la región (Correa-Cabrera, 2017; Vásquez-Galán y Corrales-Corrales, 2017). Vásquez-Galán y CorralesCorrales (2017) obtuvieron que precisamente Tamaulipas, entre el 2008 y el 2012, fue la entidad de la región noreste del país con mayor crecimiento del índice delictivo, probablemente debido al aumento de la intensidad del conflicto bélico entre grupos del crimen organizado y el Estado mexicano, además de que se incrementó la pobreza extrema, el número de personas con ingresos menores al nivel de bienestar y aumento del rezago social. Correa-Cabrera (2017) documentó que, por encima de otros 
factores como migración, desarrollo de la industria maquiladora, comercio internacional y producción de hidrocarburos, el elevado nivel de desigualdades económicas en las ciudades fronterizas de México fue probablemente el factor más determinante en el incremento del crimen.

\section{Contexto del área de estudio}

El municipio de Victoria es la capital del estado de Tamaulipas (Figura 1), para el 2010, año de la base de datos (INEGI, 2010b), contaba con 16,798 habitantes en localidades rurales y 305,155 habitantes en el ámbito urbano.

Figura 1

Mapa de localización del municipio de Victoria.
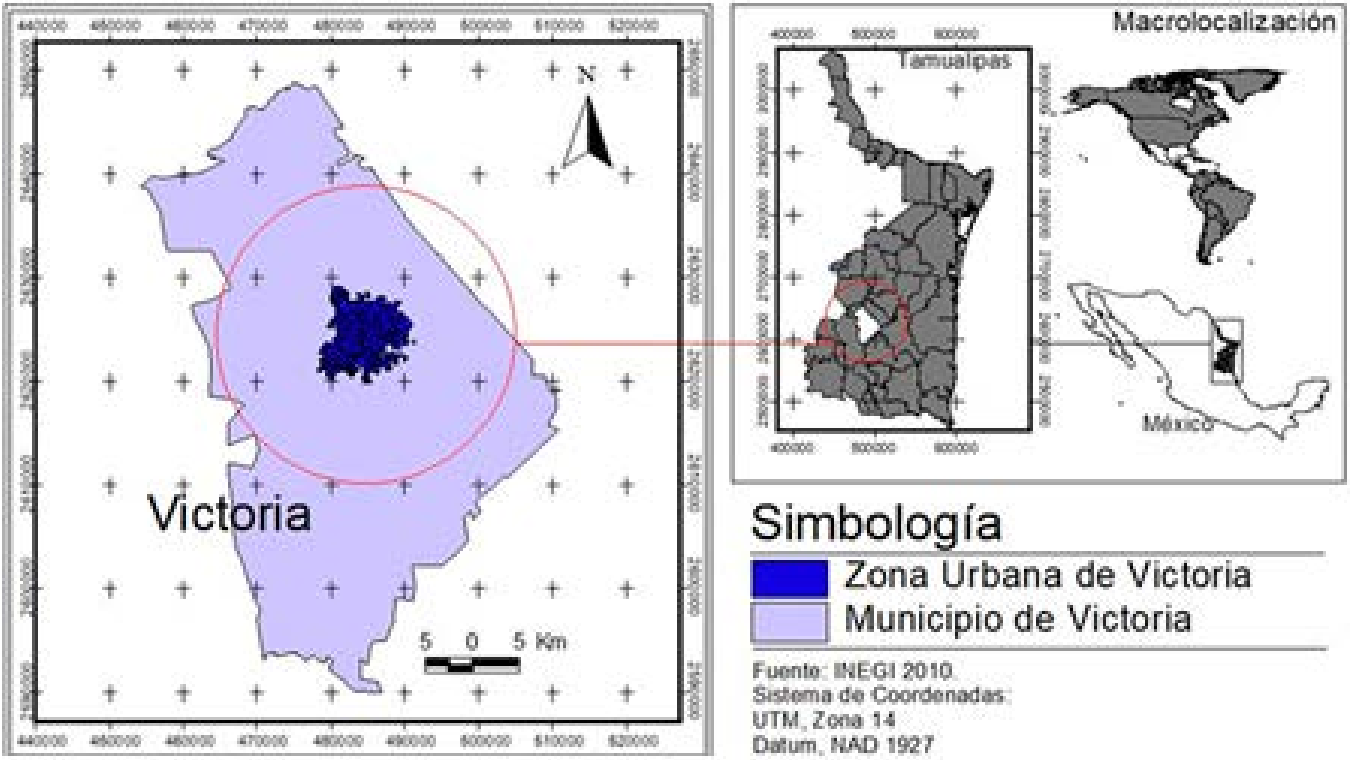

Fuente: Elaboración propia con base en INEGI 2010

Otra particularidad del municipio es que, dentro del ranking de las 50 ciudades más violentas del mundo que emite el Consejo Ciudadano para la Seguridad, Justicia y Paz (CCSPJP, 2018), Victoria ha estado en 8 de los 9 reportes publicados, ocupando los puestos más altos en 2016, cuando la ciudad fue catalogada como la $5^{\text {a }}$ ciudad más violenta del mundo, con una tasa de 84.6 homicidios por cada 100 mil habitantes. En el año 2017, obtuvo el $8^{\circ}$ lugar con una tasa de 83.32 delitos y en el año 2018, ostentó el $4 .^{\circ}$ lugar con una tasa de 86 homicidios. Victoria no aparece en el ranking del 2011, el año más cercano al contexto del presente estudio, por lo que se interpreta que la expansión de la violencia en Victoria no eclosionó hasta después de 2012.

Otro aspecto es que según datos del Consejo Nacional de Evaluación de la Política de Desarrollo Social (CONEVAL, 2010), la desigualdad en el municipio de Victoria, respecto a otras ciudades del estado, es muy alta en el año 2010, como se observa en la Figura 2. 
Figura 2

Índice de Gini municipal comparado con el Índice de Gini nacional y la media municipal.

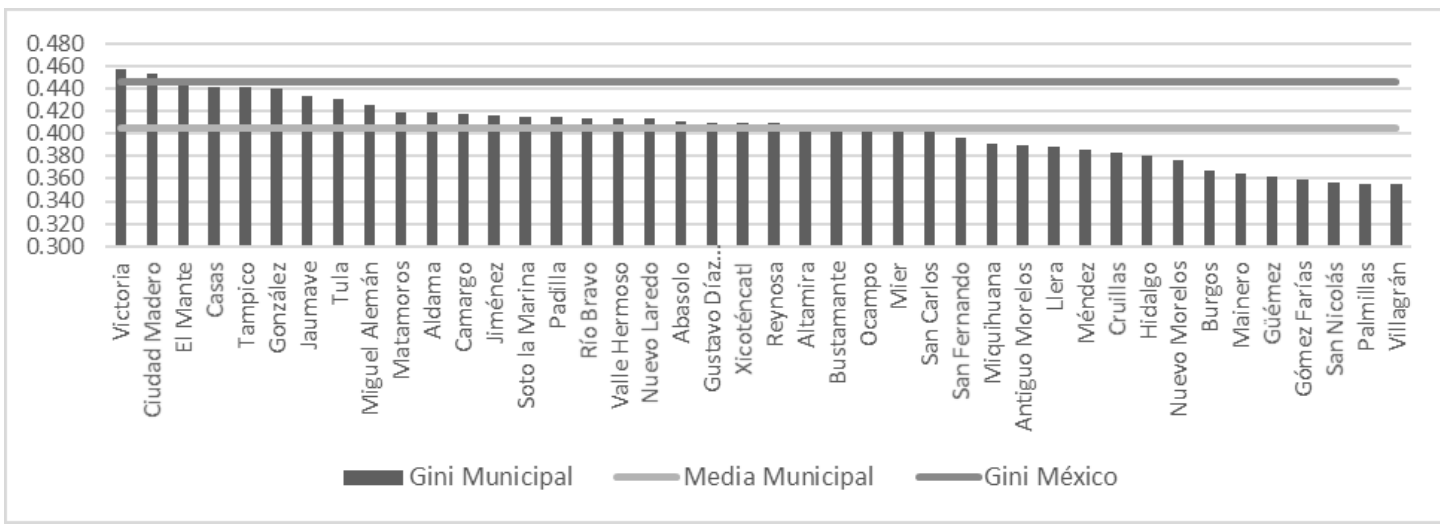

Fuente: Elaboración propia con base en CONEVAL 2010 y OCDE 2018

Lo anterior brinda una oportunidad para profundizar en el análisis de la desigualdad y el crimen en el contexto local, debido a que el municipio cuenta con una alta desigualdad en el periodo en cuestión que es previo a la explosión de la violencia y del crimen en general.

\section{Datos y metodología}

Para la obtención de los datos se recurrió a la cartografía de fuentes oficiales, con esto se pudo asignar valores a determinadas unidades territoriales e instrumentar el modelo estadístico para responder a las preguntas de investigación. A continuación, se describen la unidad de análisis de estudio y el proceso de construcción de las variables:

1. La unidad de análisis de este trabajo son las Áreas Geoestadísticas Básicas (AGEB) del municipio de Victoria, Tamaulipas, definidas por el Instituto Nacional de Geografía y Estadística (INEGI, 2010a). Estas constituyen un elemento desagregado de la unidad municipal y el universo de estudio del presente documento.

2. De acuerdo con el INEGI (2010), Ciudad Victoria está dividida en 182 AGEB (Figura 1). Estas AGEB se subdividen en 5,409 manzanas. En este caso, el AGEB, es considerada como un individuo. Cada individuo cuenta con características que determinan su diferenciación entre sus pares. Para el caso del presente trabajo, está diferenciación entre las AGEB va a ser determinada por las características contenidas en las manzanas. Es necesario mencionar que, para poder realizar el análisis estadístico, se eliminaron las AGEB que no reportaban población o que contaban con datos incompletos, eliminando 10 con estas características. En segundo lugar, se detectaron y eliminaron 2 valores atípicos univariantes. Y por último se eliminaron 4 valores atípicos multivariantes, quedando 166 AGEB consideradas para el análisis. 
Figura 3

\section{AGEB en el municipio de Victoria}

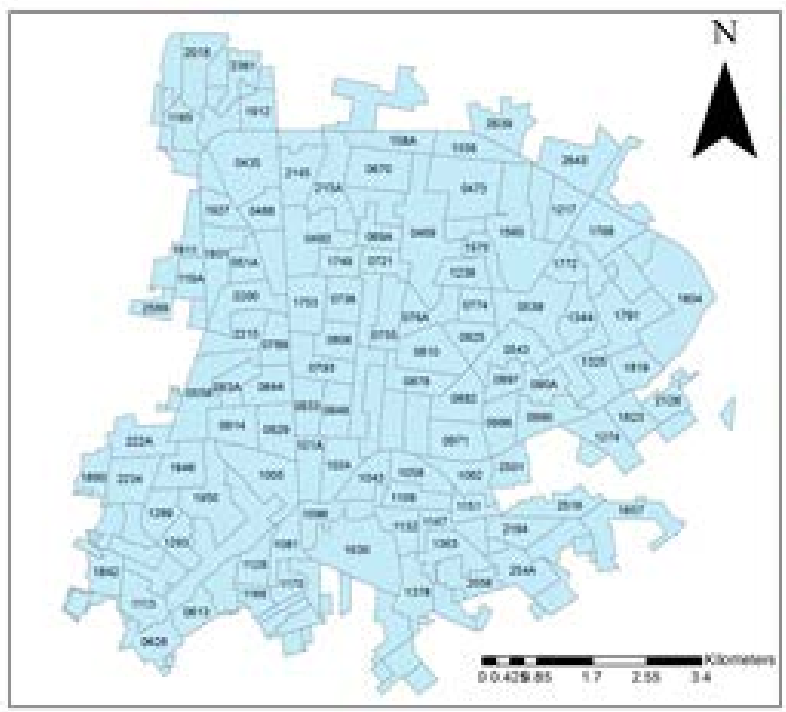

\section{Simbología}

Agebs

Fuente: Elaboración propia a partir de INEGI (2010).

Considerar el análisis a este nivel cuenta con la ventaja de poder tener una comprensión de las dinámicas que se presentan en una escala local. Se utiliza el Censo de Población (INEGI, 2010), debido a las características de la información disponible en una escala tan desagregada como lo es el AGEB y la manzana, permite localizar aspectos socioeconómicos y vincularlos a un espacio geográfico. A continuación, se describen las variables construidas para realizar el análisis, el tratamiento y características de los datos, así como la metodología utilizada para realizar el ejercicio.

\subsection{Desigualdad}

Para construir la variable de desigualdad se tomó como base y se adaptó a los indicadores disponibles la propuesta metodológica de McKenzie (2005), quien desarrolla una medición de desigualdad con variables proxis, construidas a partir del Método de Reducción de Componentes Principales (PCA, por sus siglas en inglés: Principal Component Analysis), con información de bienes de consumo, reportados en encuestas nacionales y censos de población. Como se señala en los trabajos de Filmer y Pritchett (1999) y Filmer y Pritchett (2007), estos bienes constituyen un buen indicador proxi de bienestar, y pueden funcionar tan bien como una medición tradicional. Partiendo de este proxi, se pretende medir la brecha de desigualdad entre las manzanas o cuadras, que cuentan con mayor puntaje en este indicador y las que arrojan resultados inferiores. De esta manera, intentamos reflejar el nivel de vida y las distancias sociales de la población que vive en las áreas urbanas, lo cual representa el interés principal del estudio.

Dado que el presente estudio considera al AGEB como la unidad de análisis, no es posible utilizar datos de encuestas nacionales. De tal forma que la información se ajusta a lo disponible en el Censo 
(INEGI, 2010). En la Tabla 1, se pueden observar algunas variables propuestas de D. J. McKenzie (2005) y las variables disponibles en el Censo.

\begin{tabular}{|c|c|}
\hline Variables propuestas en McKenzie (2005) & $\begin{array}{c}\text { Disponibilidad en el Censo } \\
\text { INEGI (2010) }\end{array}$ \\
\hline Número de cuartos/miembros & Sí \\
\hline Techo de ladrillo o cemento & No \\
\hline Piso de tierra & Sí \\
\hline Piso de madera o cerámica & Sí \\
\hline Agua potable & Sí \\
\hline Drenaje & Sí \\
\hline Electricidad & Sí \\
\hline Teléfono & Sí \\
\hline Automóvil & Sí \\
\hline Van & No \\
\hline Radio & Sí \\
\hline Televisión & Sí \\
\hline Máquina de cocer & No \\
\hline Estufa & No \\
\hline Refrigerador & Sí \\
\hline Lavadora & Sí \\
\hline Estéreo & No \\
\hline Propiedad urbana/ Propiedad rural & No \\
\hline Ejido o propiedad comunitaria & No \\
\hline Jefe de familia completó la primaria & No \\
\hline Jefe de familia tiene de 7-9 años de escolaridad & No \\
\hline Jefe tiene de 10-12 años de escolaridad & No \\
\hline Jefe tiene más de 12 años de escolaridad & No \\
\hline
\end{tabular}

Fuente: Elaboración propia a partir de INEGI 2010 y McKenzie y Rapoport (2005)

Es importante no pasar por alto que para identificar la desigualdad de las AGEB urbanas, es necesario desagregarlas en las unidades que las componen, en este caso la manzana urbana. De tal manera que el primer paso fue identificar el índice de bienestar para cada manzana y después, mediante el cálculo del coeficiente de Gini, se obtiene el nivel de desigualdad en relación con el nivel de bienestar de las manzanas.

Esta consideración, de utilizar a la manzana, reduce aún más los datos disponibles para este fin, debido a la ausencia de datos o a políticas de privacidad del INEGI. Lo anterior aparenta ser un problema; sin embargo, los insumos para la construcción del índice de activos a partir de PCA, no son necesariamente fijos. En la literatura varían según la disponibilidad de datos, como se puede ver en la revisión elaborada por Kolenikov y Angeles (2009). Conforme con lo anterior, se identificaron 2 dimensiones para elaborar el índice de bienestar: i) la disponibilidad de bienes; y ii) las características 
de la vivienda, que son utilizadas en la literatura consultada (Filmer y Pritchett, 1999; Montgomery et al., 2000; Vyas y Kumaranayake, 2006; Tarozzi y Deaton, 2009; Wittenberg y Leibbrandt, 2017). Las dimensiones y variables consideradas se observan en la Tabla 2.

Tabla 2 Dimensiones y variables consideradas del Censo INEGI, 2010

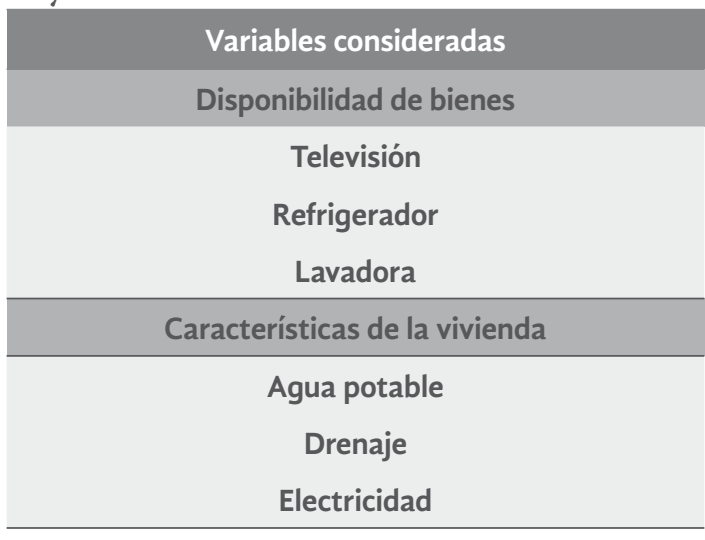

Fuente: Elaboración propia a partir de INEGI 2010

Para un mejor rendimiento en los resultados del PCA, se estandarizaron las variables considerando el tamaño poblacional de cada manzana, creando indicadores para cada variable. Una vez obtenidos los indicadores, se utilizaron como el insumo final para la aplicación del PCA. El ejercicio arrojó una variable nueva, la cual se interpreta como el índice de bienestar. Después, con este dato, se agruparon a las manzanas por AGEB para el cálculo del coeficiente de Gini. La obtención de la desigualdad por AGEB se describe en los siguientes pasos:

Obtener variables por manzana para la zona urbana del municipio de Victoria

Estandarizar variables a partir de la construcción de indicadores de vivienda y de bienes de consumo, como se presentan en las siguientes fórmulas:

$$
\begin{aligned}
& \text { IAgua Potable }=\frac{\text { Viviendas con agua potable en la Manzanas en el AGEB }}{\text { Total de Viviendas en Manzana del AGEB }} \\
& \text { IElectricidad }=\frac{\text { Viviendas con electricidad en la Manzanas en el AGEB }}{\text { Total de Viviendas en la Manzana del AGEB }} \\
& \text { IDrenaje }=\frac{\text { Viviendas con drenaje en la Manzana en el AGEB }}{\text { Total de Viviendas en la Manzana del AGEB }} \\
& \text { ITelevisió } n=\frac{\text { Número de Televisiones en la Manzana en el AGEB }}{\text { Total de Viviendas en Manzana del AGEB }} \\
& \text { IRefrigerador }=\frac{\text { Número de Televisiones en la Manzana en el AGEB }}{\text { Total de Viviendas en Manzana del AGEB }} \\
& \text { ILavadora }=\frac{\text { Número de Televisiones en la Manzana en el AGEB }}{\text { Total de Viviendas en Manzana del AGEB }}
\end{aligned}
$$


Obtener factores aplicando PCA por manzana, tomando en cuenta los resultados de los indicadores arriba expresados.

Aplicar Gini considerando los resultados del índice de bienestar por manzana que compone cada AGEB. Debido a que algunos de los factores derivados del PCA presentan resultados en valores negativos, lo cual supone una violación en los supuestos del cálculo del coeficiente de Gini, se optó por usar la propuesta de Raffinetti et al. (2015) para calcular el coeficiente con valores negativos. La fórmula se describe de la siguiente manera (Raffinetti et al., 2017):

$$
G=\frac{\Delta Y}{2 \mu Y}=\frac{1}{2 \mu y N^{2}} \sum_{i=1}^{H} \sum_{j=1}^{H}\left|y_{i}-y_{j}\right| P_{i} P_{j}
$$

Donde:

Y es el vector del índice de bienestar (incluidos valores negativos)

$\mathrm{H}$ es el número total de unidades consideradas de bienestar

Pi y Pj son cargas asociadas con yi y yj

$\mu$ y es el término de normalización que corresponde a la siguiente ecuación:

$\mu Y=\frac{\left(T_{Y}^{+}+T_{Y}^{-}\right)}{N}$

Donde:

$T_{Y}^{+} T_{Y}^{+}$es igual al promedio de todos los valores positivos

$T_{Y}^{-} T_{Y}^{-}$es igual al promedio de todos los valores negativos

El cálculo de Gini se realizó con el programa estadístico R V. 3.5.3, usando el paquete "GiniWegNegpackage", arrojando valores entre 0 y 1 , significando que entre más próximo se está de la unidad mayor desigualdad y viceversa.

Asignar a cada AGEB la intensidad de desigualdad observada.

Al Aplicar PCA a los índices señalados, resalta que los componentes se correlacionan bien entre sí. Se confirma al extraer un sólo componente que comparte el $87 \%$ de la varianza (Tabla 3). Este componente generó una variable nueva que se le asignó a cada manzana de los AGEB.

Al aplicar la prueba Kaiser-Meyer-Olkin, se obtuvo un valor de adecuación de muestra de 0.921; lo cual indica que es adecuada. En lo que respecta a la prueba de esfericidad de Bartlett, el valor es significativo $(\mathrm{P}<0.00)$.

Una vez realizado lo anterior, la variable que se genera del PCA se interpretará como la variable de bienestar. Esta se le asigna a cada una de las manzanas del municipio. En la Figura 4 se puede apreciar cómo está representado el mapa de Ciudad Victoria, según el bienestar en cada manzana. 
- Revista de Ciencias Sociales y Humanidades. ISSN-P: 0188-9834 ISSN-E:2395-8669.

Figura 4

Distribución espacial del Índice de Bienestar por manzana en Victoria 2010

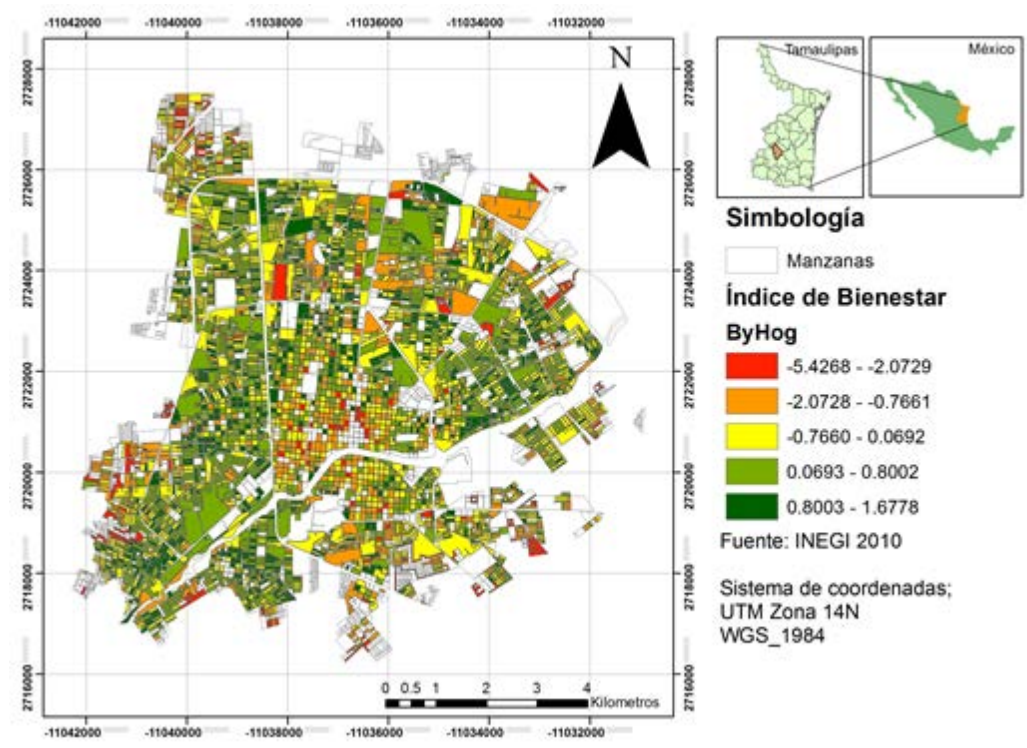

Fuente: Elaboración propia a partir de INEGI (2010).

Las manzanas catalogadas se agrupan a su respectivo AGEB, aplicando el coeficiente de Gini para medir la intensidad de la desigualdad en cada manzana, y generando así un Gini por AGEB basado en la desigualdad. El resultado está representado espacialmente en la Figura 5.

Figura 5

Desigualdad por AGEB en Victoria 2010

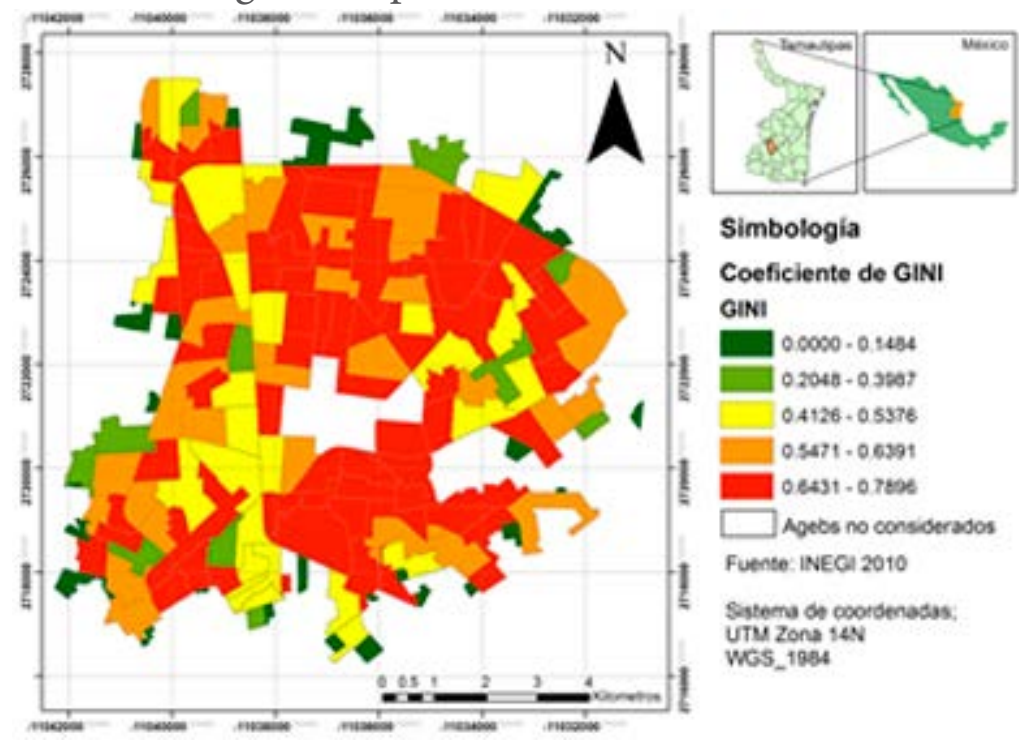

Fuente: Elaboración propia a partir de INEGI (2010). 


\subsection{Crimen}

Para obtener la variable de delitos, el estudio se ajustó a la disponibilidad de datos, debido a que la intención del trabajo no sólo es la observación del fenómeno, sino la posibilidad de usar otras técnicas para abordarlo. Los delitos contemplados sólo son del orden común, por lo que crímenes asociados al orden federal, como los relacionados con el narcotráfico, trata de personas, robo de hidrocarburos, entre otros, no se consideran. A partir de los datos provenientes de la Procuraduría General de Justicia de Tamaulipas del año 2010, se construyó una base de datos con 50 delitos para el municipio de Victoria (tabla 3).

Tabla 3

Lista de delitos

\begin{tabular}{|c|c|}
\hline Delitos & \\
\hline Abandono de obligaciones alimenticias & Homicidio culposo (suicidio) \\
\hline Abandono de personas & Impudicia \\
\hline Abuso de autoridad & Lesiones \\
\hline Abuso de confianza & $\begin{array}{l}\text { Privación ilegal de la libertad y otras ga- } \\
\text { rantías }\end{array}$ \\
\hline Allanamiento de morada & $\begin{array}{l}\text { Responsabilidad médica, técnica y adminis- } \\
\text { trativa }\end{array}$ \\
\hline Amenazas & Robo a bancos \\
\hline Armas prohibidas & Robo a comercios \\
\hline Ataques a los medios de transporte y vías de comunicación & Robo a escuelas \\
\hline Corrupción de menores & Robo a gasolineras \\
\hline $\begin{array}{l}\text { Corrupción, pornografía infantil y prostitución sexual de menores e } \\
\text { incapaces }\end{array}$ & Robo a lugar cerrado \\
\hline Daño en propiedad & Robo a tiendas de autoservicio \\
\hline Delitos de abogados, patronos y litigantes & Robo a transeúnte \\
\hline Desobediencia, resistencia y coacción de particulares & Robo con violencia \\
\hline Despojo de cosas inmuebles & Robo de semovientes menor (abigeato) \\
\hline Ejercicio abusivo de funciones & Robo de vehículos \\
\hline Estupro & Robo domiciliario \\
\hline Expendio ilícito de bebidas alcohólicas & Robo simple \\
\hline Extorsión & Secuestro \\
\hline Falsedad en declaraciones y en informes dados a una autoridad & Sustracción de menores por los padres \\
\hline Falsificación y circulación de títulos y documentos de crédito público & Tentativa de robo \\
\hline Falsificación y uso de documentos públicos y privados & Tentativa de violación \\
\hline Fraude & Usura \\
\hline Golpes y violencias físicas simples & Violación \\
\hline Homicidio & Violencia intrafamiliar \\
\hline
\end{tabular}

Fuente Elaboración propia a partir de base de datos de PGJ (2010). 
En el presente estudio sólo se consideran 19 de estos delitos que, de acuerdo con la literatura consultada, cuentan con alguna asociación con la variable de desigualdad, como se muestra en la tabla 4. La información consultada para construir la base de datos contempla: tipo de delito, año del delito y las coordenadas de longitud y latitud, con lo que se permitió la georreferenciación de los delitos por AGEB(Arc View GIS V.3.2).

Tabla 4

Delitos seleccionados, estudios donde se asocian a factores sociales y frecuencia en el municipio.

\begin{tabular}{|c|c|c|}
\hline $\begin{array}{l}\text { Estudios que relacionan con } \\
\text { factores sociales }\end{array}$ & Tipo de delito & $\begin{array}{l}\text { Frecuencia } \\
\text { de delitos } \\
\text { en el muni- } \\
\text { cipio }\end{array}$ \\
\hline (Hodges, 2020) & Abandono de obligaciones alimenticias & 157 \\
\hline \multirow{6}{*}{$\begin{array}{l}\text { (Mui y Ali, 1997),Kelly } \\
\text { (2000), Neumayer (2005); } \\
\text { Coccia (2017), (Enamorado } \\
\text { et al., 2016) }\end{array}$} & Daño en propiedad & 189 \\
\hline & Amenazas & 108 \\
\hline & Homicidio & 25 \\
\hline & Lesiones & 289 \\
\hline & Robo con violencia & 5 \\
\hline & Golpes y violencia física simple & 55 \\
\hline (Bateset al., 2004) & Violencia intrafamiliar & 121 \\
\hline \multirow{3}{*}{ Mui and Ali (1997) } & Abuso de confianza & 53 \\
\hline & Fraude & 133 \\
\hline & Falsedad en declaraciones y en informes dados a una autoridad & 11 \\
\hline \multirow{9}{*}{$\begin{array}{l}\text { (Mui y Ali, 1997), Kelly } \\
\text { (2000), Neumayer (2005); } \\
\text { Coccia (2017) }\end{array}$} & Robo a comercios & 62 \\
\hline & Robo a escuelas & 22 \\
\hline & Robo a lugar cerrado & 48 \\
\hline & Robo de vehículos & 363 \\
\hline & Robo domiciliario & 393 \\
\hline & Robo simple & 655 \\
\hline & Tentativa de robo & 16 \\
\hline & Otros delitos & 54 \\
\hline & Total & 2759 \\
\hline
\end{tabular}

Fuente: Elaboración propia a partir de la PGJ (2010). 
- Revista de Ciencias Sociales y Humanidades. ISSN-P: 0188-9834 ISSN-E:2395-8669.

La suma de los delitos se agrupa en las respectivas AGEB de incidencia, generando así diferencias individuales observables. Para normalizar las cantidades en función de la población de cada AGEB, se calculó la tasa de delitos por cada 100 habitantes, la fórmula se aplicó de la siguiente manera:

$$
T D e l=\left(\frac{D A g e b}{\text { PobAgeb }}\right) * 10 T \text { Del }=\left(\frac{\text { DAgeb }}{\text { PobAgeb }}\right) * 100
$$

Donde:

TDel es la tasa de delitos

DAGEB es el total de delitos dentro del AGEB

PobAGEB es la población del AGEB

Una vez obtenidas las tasas de delitos para cada AGEB, es posible visualizar la representación espacial de los delitos en Victoria (Figura 6).

Figura 6

Tasa de delitos por cada 100 habitantes en AGEB de Victoria 2010

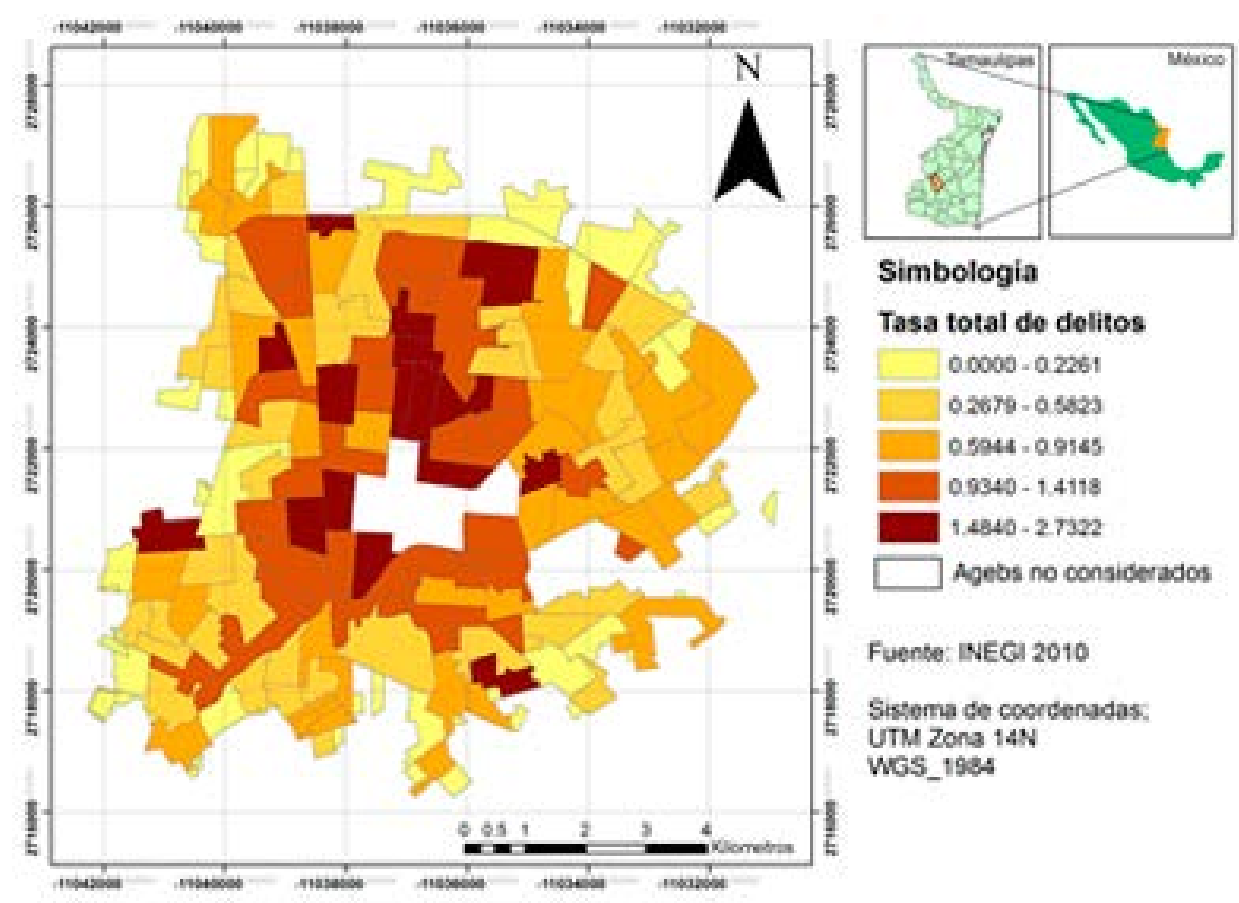

Fuente: Elaboración propia a partir de INEGI (2010). 


\subsection{Modelo}

Se asume que la desigualdad tiene la capacidad de predecir la cantidad de delitos en las AGEB. De tal manera que se plantea una regresión lineal simple para comprobarlo.

Como se mencionó anteriormente, las dos variables en cuestión son construcciones previas. La variable independiente de la desigualdad por AGEB fue construida a partir de proxis y generada con PCA. La variable dependiente de delitos fue el resultado de la suma de las infracciones. En ese orden, al contar con estas variables es posible usar la prueba estadística de regresión lineal simple, expresada de la siguiente manera:

$$
Y_{1}=b_{0}+b_{1} X 1_{i}+\varepsilon_{1}
$$

Donde:

Y es el número de delitos

b1 es el coeficiente o parámetro del índice de Gini

Xi es Coeficiente de Gini

عi es el error

b0 es la constante

\section{Resultados y hallazgos}

$\mathrm{Al}$ aplicarse el modelo de regresión lineal, se obtuvo como resultado la existencia de una correlación positiva entre desigualdad y delitos $(r=0.510)$. Esta relación es significativa $(P>0.001)$, por lo que se identifica que a mayor desigualdad corresponde, en parte, una mayor tasa de delitos por cada

100 habitantes. Asimismo, se obtuvo que el índice de Gini explica la varianza en la tasa de delitos $\left(r^{\wedge} 2=0.26\right)$ como se observa en la Figura 7 , lo cual significa que la desigualdad explica el $26 \%$ de la variación en delitos. 
Figura 7

Correlación entre Tasa de delitos e índice de Gini

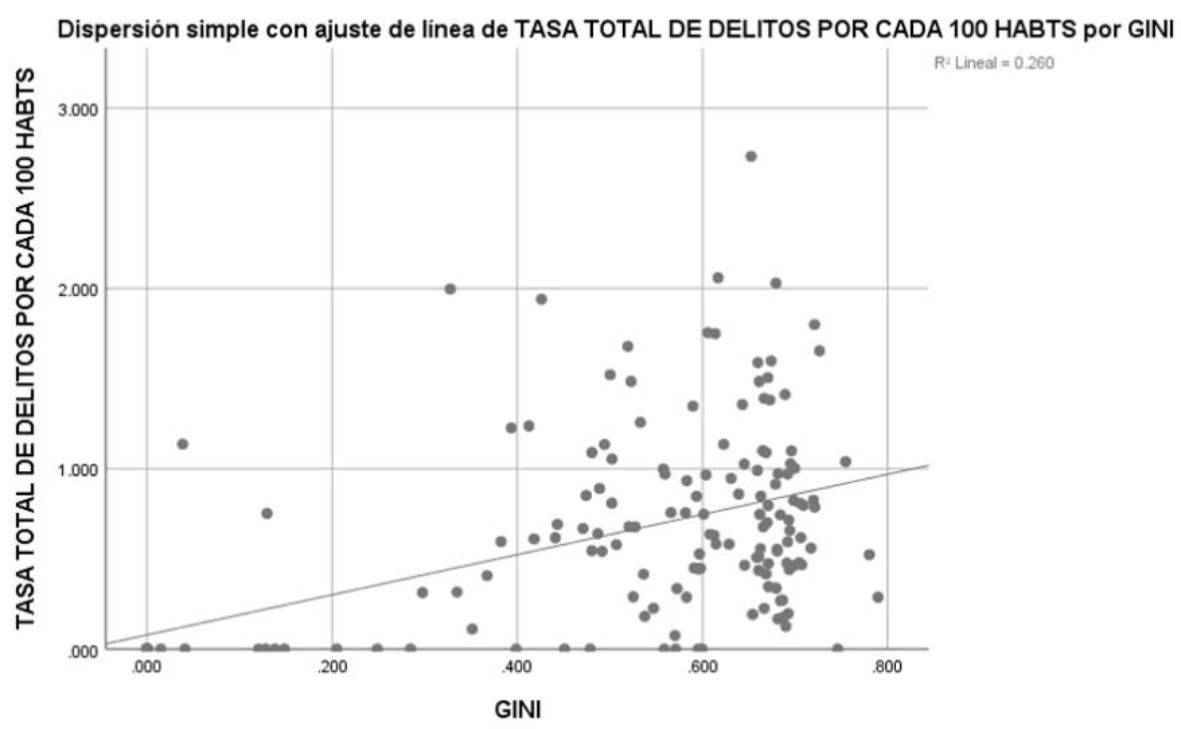

Fuente: Elaboración propia a partir de INEGI (2010).

De un mismo modo, se observa en la Tabla 5, un valor de beta del Gini de $(\mathrm{b}=1.114)$, el cual predice que por cada punto porcentual que aumenta el coeficiente de Gini, se traduce en un aumento en la tasa de 1.11 delitos por cada 100 habitantes en las AGEB del municipio. Puesto en contexto, una AGEB que presente aumento de desigualdad, corresponderá un aumento en la tasa de crimen.

Tabla 5

Coeficientes entre delitos e índice de Gini en Victoria 2010

\begin{tabular}{|c|c|c|c|c|c|c|c|c|}
\hline & \multirow{2}{*}{ Modelo } & \multicolumn{2}{|c|}{$\begin{array}{c}\text { Coeficientes no estan- } \\
\text { darizados }\end{array}$} & \multirow{2}{*}{$\begin{array}{c}\text { Coeficientes } \\
\text { estandarizados } \\
\text { Beta }\end{array}$} & \multirow{2}{*}{$\mathrm{t}$} & \multirow{2}{*}{ Sig. } & \multicolumn{2}{|c|}{$\begin{array}{l}\text { Estadísticas de coli- } \\
\text { nealidad }\end{array}$} \\
\hline & & B & $\begin{array}{l}\text { Desv. } \\
\text { Error }\end{array}$ & & & & Tolerancia & VIF \\
\hline \multirow{2}{*}{1} & (Constante) & 0.078 & 0.079 & & 0.993 & 0.322 & & \\
\hline & GINI & 1.114 & 0.145 & 0.510 & 7.656 & 0.000 & 1.000 & 1.000 \\
\hline
\end{tabular}

Fuente: elaboración propia. 
- Revista de Ciencias Sociales y Humanidades. ISSN-P: 0188-9834 ISSN-E:2395-8669.

Para observar y dimensionar distintos efectos, se han calculado correlaciones de la población y los elementos que componen la tasa de delitos. En ese sentido, vemos que la relación en ambos casos es significativa, con una correlación de $(\mathrm{r}=0.510)$ y $(\mathrm{r}=0.520)$ respectivamente (Tabla 6). El coeficiente de Gini se asocia positivamente a la población, por lo que se constata que mientras más población, mayor la desigualdad. Sin embargo, por las densidades de población de los AGEB, esto no debería sorprender, pues cuando más personas haya en una demarcación territorial, siempre habrá mayor probabilidad de ocurrencia de un fenómeno. Con esta lógica, también podrían asociarse otras variables, como jefatura femenina, escolaridad o propensión al voto, entre otras.

Tabla 6

Correlaciones entre índice de Gini y tipo de delito

\begin{tabular}{|c|c|c|}
\hline & & \\
\hline Variables & $\begin{array}{l}\text { Correlación de Pear- } \\
\text { son }\end{array}$ & Sig. (bilateral) \\
\hline Abandono de obligaciones alimenticias & $.325 * *$ & 0.000 \\
\hline Abuso de confianza & $.194^{*}$ & 0.012 \\
\hline Amenazas & $.302 * *$ & 0.000 \\
\hline Daño en propiedad & $.319 * *$ & 0.000 \\
\hline Otros & $.262 * *$ & 0.001 \\
\hline Falsedad en declaraciones y en informes dados a una autoridad & 0.129 & 0.096 \\
\hline Fraude & $.302 * *$ & 0.000 \\
\hline Golpes y violencias físicas simples & 0.103 & 0.182 \\
\hline Homicidio & $.160 *$ & 0.038 \\
\hline Lesiones & $.359 * *$ & 0.000 \\
\hline Robo a comercios & 0.124 & 0.109 \\
\hline Robo a escuelas & 0.077 & 0.319 \\
\hline Robo a lugar cerrado & 0.136 & 0.078 \\
\hline Robo con violencia & 0.034 & 0.657 \\
\hline Robo de vehículos & $.298 * *$ & 0.000 \\
\hline Robo domiciliario & $.373 * *$ & 0.000 \\
\hline Robo simple & $.332 * *$ & 0.000 \\
\hline Tentativa de robo & $.154^{*}$ & 0.045 \\
\hline Violencia intrafamiliar & $.176 *$ & 0.022 \\
\hline Población & $.520 * *$ & 0.000 \\
\hline Tasa total de delitos & $.510 * *$ & 0.000 \\
\hline
\end{tabular}

Fuente: Elaboración propia a partir de datos de INEGI (2010). 
Resulta de interés observar como no todos los delitos guardan una correlación significativa. Delitos como falsedad en declaraciones y en informes dados a una autoridad, lesiones, robo a comercios, robo a escuelas, robo a lugar cerrado y robo con violencia, en el modelo presentado, parecen no guardar relación con el fenómeno de desigualdad.

Los hallazgos del trabajo contribuyen a la evidencia que sostiene que existe una relación positiva entre desigualdad y crimen (Lee et al., 2014; Enamorado et al., 2016; Coccia, 2017; Costantini, Meco y Paradiso, 2018).

El análisis realizado a una escala urbana con un nivel alto de desagregación, como lo es el AGEB, permite observar variaciones en crímenes y grados de desigualdad entre distintas zonas del espacio urbano. En ese sentido, el presente estudio aporta elementos descriptivos para comprender el fenómeno desde un punto de vista espacial.

Investigaciones como la de Chamberlain y Hipp (2015), identificaron en vecindarios de Estados Unidos una relación positiva entre la desigualdad y el crimen, en función de la proximidad con vecindarios económicamente más desaventajados. En un mismo plano, Metz y Burdina (2018), encontraron que la desigualdad entre las manzanas o cuadras de una ciudad tienen un papel fundamental en determinar el nivel de crimen de propiedad, siendo que mientras más se extiende la brecha de desigualdad entre manzanas ricas y pobres, el nivel de crimen de propiedad se incrementará. En otro sentido, Kang (2016) cuestiona los estudios de desigualdad y crimen a gran escala, debido a que no se tienen los mismos resultados en una escala a nivel condados, donde la concentración de pobreza es lo que explica crímenes violentos. La aportación de Kang invita a realizar análisis más desagregados y a detectar dinámicas que escapan a los análisis más amplios.

Los resultados del estudio son de utilidad práctica. Las fuentes de información y la metodología implementada permitieron explorar y desarrollar el análisis en unidades muy desagregadas, generando información nueva en el proceso, como fue el nivel de desigualdad. Lo anterior permite realizar análisis ulteriores, considerando otras variables y metodologías. No obstante, es pertinente señalar que el estudio cuenta con limitaciones a tomar en consideración. En primer lugar, la medición de desigualdad se basó en información secundaria, muchos datos no estaban disponibles para hacer el análisis, esto implicó una pérdida de $25 \%$ de la información de las manzanas urbanas. Por otro lado, al contemplar los delitos agregados limitó la posibilidad de observar cómo el fenómeno de la desigualdad interactúa con las diferentes modalidades de delitos.

Cabe señalar que el AGEB cuenta con una ventaja operativa para identificar y clasificar zonas urbanas de acuerdo con alguna característica. Al ser una unidad en el espacio territorial, pueden ser abordadas en términos de interacción con otras instalaciones sociales, grupos de población y así, generar más insumos de información. Empero, la forma del AGEB es irregular. Las manzanas que los componen varían en un rango amplío, desde 1 a 2 manzanas por AGEB, hasta más de 40 manzanas, lo que implica un problema de comparabilidad.

Por último, es importante señalar que estos resultados deben tomarse con cautela, debido a que son generados a partir de un ejercicio realizado en un municipio con características particulares (estado fronterizo) y en un contexto histórico especial como fue la guerra contra el crimen organizado (2010). Al realizar un ejercicio similar en el municipio de Matamoros, Tamaulipas, la relación entre crimen y desigualdad $(r=0.229)$ fue significativa con un $(P=0.001)$ y una varianza explicada de $\left(r^{\wedge} 2=0.052\right)$ 
(ver Anexo 1). Lo anterior implica que, aunque existe una relación, esta puede estar sujeta a otros factores que no fueron considerados en el modelo.

\section{Conclusión}

Los resultados obtenidos parecen ser consecuentes con los estudios descritos. El análisis arrojó tres aportaciones concernientes a lo discutido previamente.

En primer lugar, la desigualdad se midió partiendo de una escala de manzanas urbanas. Lo anterior posibilitó la medición de la intensidad de las desigualdades entre cuadras urbanas permitiendo así observar el fenómeno en ese nivel. En segundo lugar, la relación entre las 2 variables fue positiva y significativa, lo cual brinda un elemento más para la discusión sobre los orígenes del crimen, sobre todo porque en México, hasta donde se sabe, no existen estudios previos que hayan verificado esta relación a un nivel tan desagregado como el realizado en este trabajo. La tercera aportación es la ubicación geográfica de los fenómenos. El estudio señala dónde están ocurriendo estos fenómenos y a qué nivel de intensidad por AGEB. La localización territorial desagregada tiene implicaciones para el diseño y evaluación de políticas públicas, al disponer de información adicional sobre un problema que afecta la vida pública y para el cual pueden implementarse acciones muy específicas, focalizadas, que mejoren las condiciones de vida del territorio.

Los datos recopilados corresponden al año 2010. Para los autores, el análisis realizado con base en estos datos representa una oportunidad para entender la dinámica de los fenómenos de la desigualdad y el crimen. Del mismo modo, hay que tomar en consideración que en esos años se desarrollaba la guerra contra el crimen organizado, lo cual podría generar un sesgo en los resultados, al alterar la dinámica de las ciudades, pero al mismo tiempo representa un indicio de un fenómeno que todavía está por explicarse.

Por último, se abre el cuestionamiento sobre cuáles son los otros factores que pudieran ejercer influencia sobre la relación estudiada, como la pobreza, el capital social, la migración, la acción gubernamental y el desarrollo económico. Asimismo, la investigación constituye un punto de partida y deja abierta la posibilidad de estudiar otros municipios, con características similares o no.

\section{Referencias}

Bates, L. M., Schuler, S. R., Islam, F. e Islam, M. K. (2004). Socioeconomic factors and processes associated with domestic violence in rural Bangladesh. International Family Planning Perspectives, 30(4), 190-199. https://doi.org/10.1363/3019004

Becker, G. (1968). Crime and Punishment: An Economic Approach. Journal of Political Economy, 76(2), 169-217. http://www.jstor.org/stable/1830482

Blau, J. R. y Blau, P. M. (1982). The cost of inequality: Metropolitan structure and violent crime. American Sociological Review, 47(1), 114-129. https://doi.org/10.2307/2095046 
Concejo Ciudadano para la Paz y Justica A. C. (2018). Metodología del ranking 2017 de las 50 ciudades más violentas del mundo [Internet]. Ciudad de México: Seguridad, Justicia y Paz. Recuperado el 4 de agosto de 2019. http://www.seguridadjusticiaypaz.org.mx/biblioteca/download/6-prensa/242las-50-ciudades-mas-violentas-del-mundo-2017-metodologia

Chamberlain, A. W. y Hipp, J. R. (2015). It's all relative: Concentrated disadvantage within and across neighborhoods and communities, and the consequences for neighborhood crime. Journal of Criminal Justice, 43(6), 431-443. https://doi.org/10.1016/j.jcrimjus.2015.08.004

Chintrakarn, P. y Herzer, D. (2012). More inequality, more crime? A panel cointegration analysis for the United States. Economics Letters, 116(3), 389-391. https://doi.org/10.1016/j.econlet.2012.04.014

Coccia, M. (2017). A theory of general causes of violent crime: Homicides, income inequality and deficiencies of the heat hypothesis and of the model of CLASH. Aggression and Violent Behavior, 37, 190-200. https://doi.org/10.1016/j.avb.2017.10.005

Consejo Nacional de Evaluación de la Política de Desarrollo Social (CONEVAL) (2010). La cohesión social. Recuperado el 2 de octubre de 2019. https://www.coneval.org.mx/Medicion/Paginas/Cohesion_Social.aspx

Correa-Cabrera, G. (2015). Desigualdades y flujos globales en la frontera noreste de México: Los efectos de la migración, el comercio, energéticos y crimen organizado transnacional. Canadian Journal of Latin American and Caribbean Studies, 40(3), 326-350. https://doi.org/10.1080/08263663.2015.1074853

Costantini, M., Meco, I. y Paradiso, A. (2018). Do inequality, unemployment and deterrence affect crime over the long run? Regional Studies, 52(4), 558-571. https://doi.org/10.1080/00343404.2017.1341626

Ehrlich, I. (1973). Participation in Illegitimate Activities: A Theoretical and Empirical Investigation. Journal of Political Economy, 81(3), 521-565. https://doi.org/10.1086/260058

Enamorado, T., López-Calva, L. F., Rodríguez-Castelán, C. y Winkler, H. (2016). Income inequality and violent crime: Evidence from Mexico's drug war. Journal of Development Economics, 120, 128-143. https://doi.org/10.1016/j.jdeveco.2015.12.004

Fajnzylber, P., Lederman, D. y Loayza, N. (2002). Inequality and violent crime. Journal of Law and Economics, 45(1), 1-40. https://doi.org/10.1086/338347

Filmer, D. y Pritchett., L. (1999). The effect of household wealth on educational attainment: Evidence from 35 countries. Population and Development Review, 25(1), 85-120.

Filmer, D. y Pritchett, L. H. (2007). Estimating Wealth Effects without Expenditure Data-or Tears: An Application to Educational Enrollments in States of India. Demography, 38(1), 115-132. https://doi. org/10.2307/3088292

Hodges, L. (2020). Do low-income parents who receive unemployment insurance pay more child support? Children and Youth Services Review, 111, https://doi.org/10.1016/j.childyouth.2020.104834

Hope, A. (2013, 1 de noviembre) Violencia 2007-2011. La tormenta perfecta, Nexos. www.nexos.com. $\mathrm{mx} / \mathrm{p} \mathrm{p}=15547$

INEGI (2010a) Manual de cartografía geoestadística. Recuperado el 25 de agosto de 2018. https://www. inegi.org.mx/contenidos/temas/mapas/mg/metadatos/manual_cartografia_censal.pdf

INEGI (2010b) Principales resultados por AGEB y manzana urbana. Recuperado el 14 de noviembre de 2018. http://www.inegi.org.mx/est/contenidos/proyectos /ccpv/cpv2010/iter_ageb_manzana_2010.aspx 
Kang, S. (2016). Inequality and crime revisited: effects of local inequality and economic segregation on crime. Journal of Population Economics, 29(2), 593-626. https://doi.org/10.1007/s00148-015-0579-3

Kennedy, B. P., Kawachi, I., Prothrow-Stith, D., Lochner, K., y Gupta, V. (1998). Social capital, income inequality, and firearm violent crime. Social Science \& Medicine, 47(1), 7-17. https://doi.org/1016/ S0277-9536(98)00097-5

Kelly, M. (2000). Inequality and crime. Review of Economics and Statistics, 82(4), 530-539. https://doi. org/10.1162/003465300559028

Kolenikov, S. y Angeles, G. (2009). Socioeconomic status measurement with discrete proxy variables: Is principal component analysis a reliable answer? The Review of Income and Wealth, 55(1), 128-155. https://doi.org/10.1109/MC.2006.355

Lee, B. X., Wexler, B. E. y Gilligan, J. (2014). Political correlates of violent death rates in the U.S., 19002010: Longitudinal and cross-sectional analyses. Aggression and Violent Behavior, 19(6), 721-728. https://doi.org/10.1016/j.avb.2014.09.017

McKenzie, D. J. (2005). Measuring inequality with asset indicators. Journal of Population Economics, 18 (2), 229-260. https://doi.org/10.1007/s00148-005-0224-7

Merino, J. (2011, 1 de junio) Los operativos conjuntos y la tasa de homicidios: Una medición, Recuperado el 25 de agosto de 2018. Nexos. https://www.nexos.com.mx/?p=14319

Merton, R. K. (1938). Social Structure and Anomie. American Sociological Review, 3(5), 672. https://doi. org/10.2307/2084686

Metz, N. y Burdina, M. (2018). Neighbourhood income inequality and property crime. Urban Studies, 55 (1), 133-150. https://doi.org/10.1177/0042098016643914

Montgomery, M. R., Gragnolati, M., Burke, K. A. y Paredes, E. (2000). Measuring living standards with proxy variables. Demography, 37(2), 155-174. http://www.ncbi.nlm.nih.gov/pubmed/10836174

Mui, H. W. y Ali, M. M. (1997) Economic analysis of crime and punishment: an Asian case. Applied Economics Letters, 4(4), 261-265. https://doi.org/10.1080/758518507

Neumayer, E. (2005). Inequality and violent crime: Evidence from data on robbery and violent theft. Journal of Peace Research, 42(1), 101-112. https://doi.org/10.1177/0022343305049669

OECD. (2020), Income inequality [Indicador], Recuperado el 25 de agosto de 2019. https://doi. org/10.1787/459aa7f1-en

Procuraduría General de Justicia (PGJE) (28 de julio, 2010). Mapa delictivo [Fichero de datos]. Dirección de Informática de la PGJE, Ciudad Victoria, Tamaulipas, México. https://www.tamaulipas.gob.mx/ fiscalia/incidencia-delictiva-2020/

Pridemore, W. A. (2011). Poverty matters: A reassessment of the inequality-homicide relationship in crossnational studies. British Journal of Criminology, 51(5), 739-772. https://doi.org/10.1093/bjc/azr019

Pridemore, W. A. y Trent, C. L. S. (2010). Do the invariant findings of land, Mccall, and Cohen generalize to cross-national studies of social structure and homicide? Homicide Studies, 14 (3), 296-335. https://doi.org/10.1177/1088767910371184

Raffinetti, E., Siletti, E. y Vernizzi, A. (2015) On the Gini coefficient normalization when attributes with negative values are considered. Statistical Methods and Applications, 24 (3), 507-521. https://doi. org/10.1007/s10260-014-0293-4 
Raffinetti, E., Siletti, E. y Vernizzi, A. (2017). Analyzing the effects of negative and non-negative values on income inequality: evidence from the survey of household income and wealth of the Bank of Italy (2012). Social Indicators Research, 133 (1), 185-207. https://doi.org/10.1007/s11205-016-1354-x

Shaw, C. R. y McKay, H. D. (1942). Juvenile delinquency and urban areas. The University of Chicago Press. Tarozzi, A. y Deaton, A. (2009). Using census and survey data to estimate poverty and inequality for small areas. Review of Economics and Statistics, 91(4), 773-792.

Vásquez Galán, B. I. y Corrales Corrales, S. (2017). Análisis de correlación de la violencia y la criminalidad en el noreste de México entre 2008 y 2014. Sociedad y Economía, 32, 127-146. http://www.scielo.org. co/scielo.php?script=sci_abstract\&pid=S1657-63572017000100127\&lng=e\&nrm=iso\&tlng=es

Vyas, S. y Kumaranayake, L. (2006). Constructing socio-economic status indices: How to use principal components analysis. Health Policy and Planning, 21(6), 459-468. https://doi.org/10.1093/heapol/czl029

Wittenberg, M. y Leibbrandt, M. (2017). Measuring inequality by asset indices: a general approach with application to South Africa. Review of Income and Wealth, 63(4), 706-730.

https://doi.org/10.1111/roiw.12286

\section{Anexo 1: Caso de la ciudad de Matamoros}

Se realizó un ejercicio similar al expuesto en el artículo en Matamoros. Primero, se obtuvo el índice de bienestar por manzana (Figura 8). Paso seguido, se calculó el Gini para cada uno de los AGEB (Figura 9). En el proceso se eliminaron manzanas que no reportaban población y las que no tenían la información pública.

Figura 8

Distribución espacial del Índice de Bienestar por manzana en Matamoros 2010

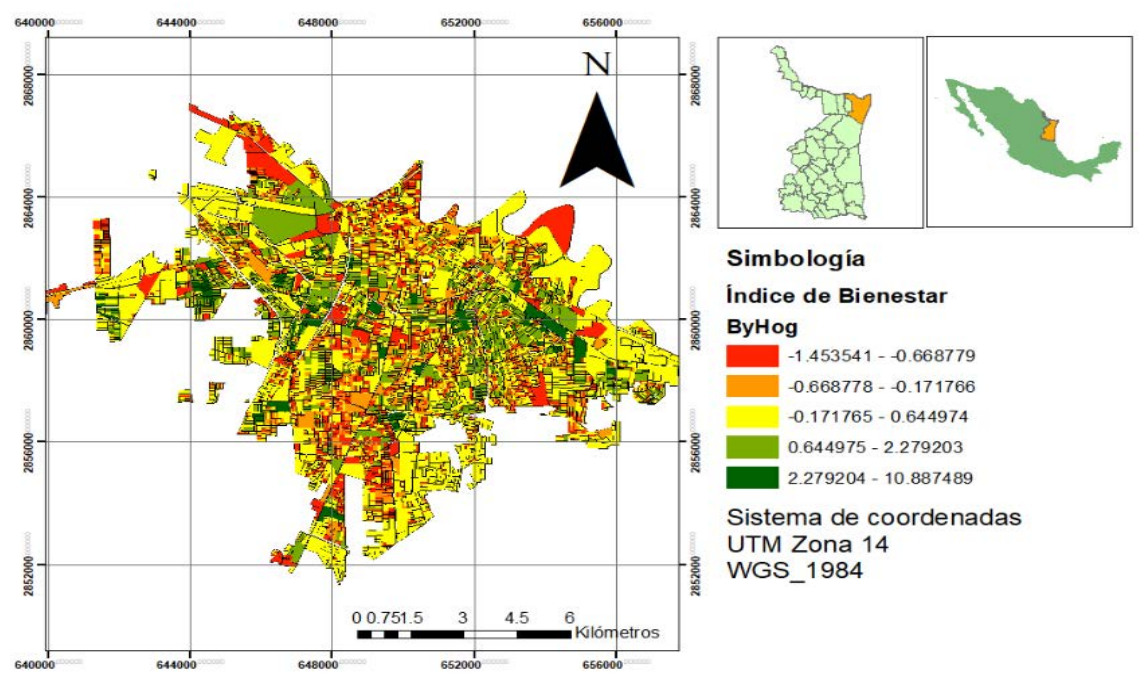

Fuente: Elaboración propia a partir de INEGI (2010). 
Figura 9

Desigualdad por AGEB en Matamoros 2010

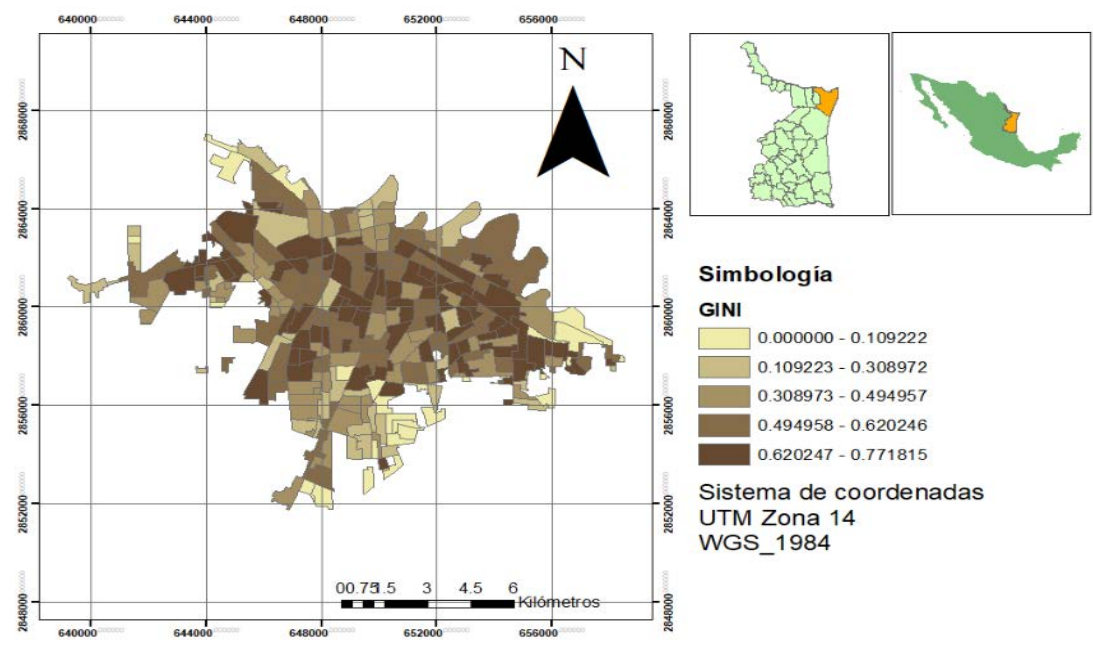

Fuente: Elaboración propia a partir de INEGI (2010).

Para obtener la tasa de delitos, se realizó un geoprocesamiento en el programa Arc View GIS V.3.2, con lo cual se asignaron los delitos a cada uno de las AGEB. En el análisis se eliminaron AGEBs sin población, así mismo 2 AGEBs que mostraban valores atípicos univariante y 2 multivariantes.

Figura 10

Tasa de delitos por cada 100 habitantes en AGEB de Matamoros 2010

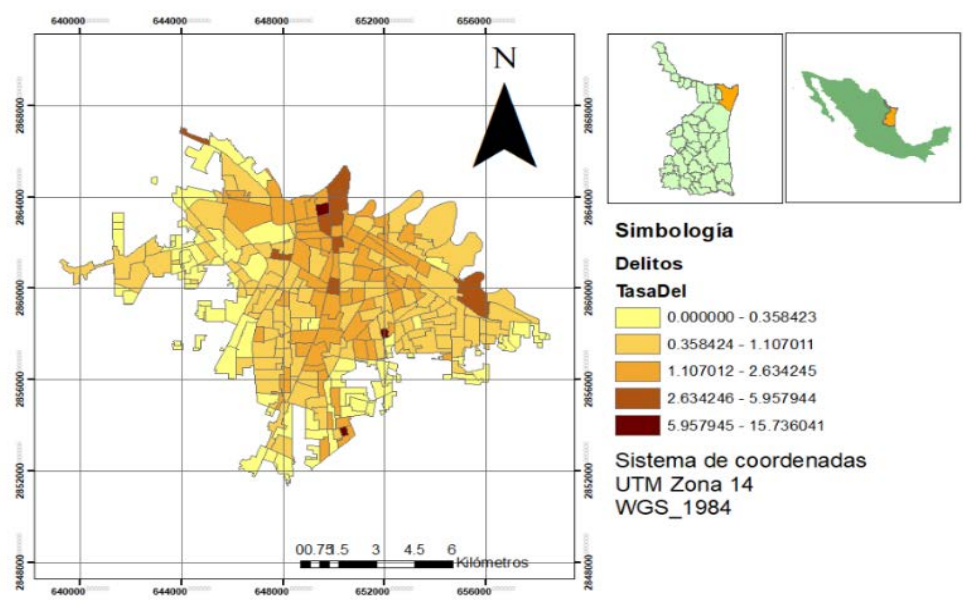

Fuente: Elaboración propia a partir de INEGI (2010). 
Al realizar un análisis de regresión lineal, se obtuvo como resultado la existencia de una correlación positiva entre desigualdad y delitos $(r=0.229)$. Esta relación es significativa $(P>0.001)$. Asimismo, se obtuvo que el índice de Gini explica la varianza de forma débil en la tasa de delitos ( $\left.\mathrm{r}^{\wedge} 2=0.052\right)$ como se observa en la Figura 11, lo cual significa que la desigualdad explica el $5.2 \%$ de la variación en delitos.

Figura 11.

Correlación entre Tasa de delitos e índice de Gini en el municipio de Matamoros

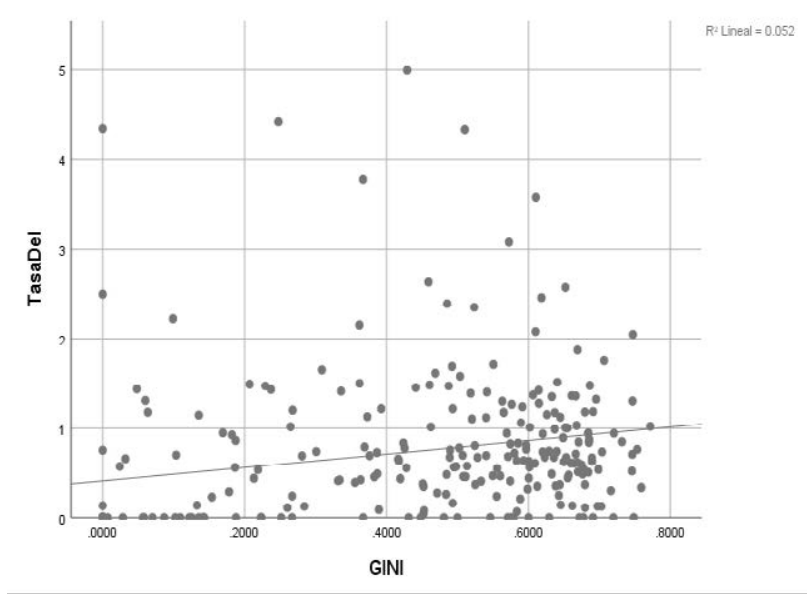

Fuente: Elaboración propia a partir de INEGI (2010).

Del mismo modo, se observa un valor de beta del Gini de $(b=0.753)$ (Tabla 7), el cual predice que por cada punto porcentual que aumenta el coeficiente de Gini, se traduce en un aumento en la tasa de 0.75 delitos por cada 100 habitantes en las AGEB del municipio. Puesto en contexto, a una AGEB que presente aumento de desigualdad, corresponderá un aumento en la tasa de crimen.

Tabla 7.

Coeficientes entre delitos e índice de Gini en Victoria 2010

\begin{tabular}{|c|c|c|c|c|c|c|c|c|c|c|c|}
\hline \multicolumn{12}{|c|}{ Coeficientes } \\
\hline & \multirow{2}{*}{ Modelo } & \multicolumn{2}{|c|}{$\begin{array}{l}\text { Coeficientes no } \\
\text { estandarizados }\end{array}$} & \multirow{2}{*}{$\begin{array}{c}\begin{array}{c}\text { Coeficientes } \\
\text { estandarizados }\end{array} \\
\text { Beta }\end{array}$} & \multirow{2}{*}{$t$} & \multirow{2}{*}{ Sig. } & \multicolumn{3}{|c|}{ Correlaciones } & \multicolumn{2}{|c|}{$\begin{array}{c}\text { Estadísticas de } \\
\text { colinealidad }\end{array}$} \\
\hline & & B & Desv. Error & & & & Orden cero & Parcial & Parte & Tolerancia & VIF \\
\hline \multirow{2}{*}{ 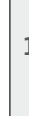 } & (Constante) & 0.412 & 0.097 & & 4.257 & 0.000 & & & & & \\
\hline & Gini & 0.753 & 0.198 & 0.229 & 3.806 & 0.000 & 0.229 & 0.229 & 0.229 & 1.000 & 1.000 \\
\hline
\end{tabular}

